survival or the incidence of acute cellular rejection and biliary anastomotic strictures.

Disclosure of Interest None Declared.

\section{PWE-142 IS LIVER DISEASE OVERLOOKED IN PATIENTS WITH PSORIASIS?}

1,2J Ooi*, ${ }^{1,3}$ S Chinthapalli, ${ }^{1,3} \mathrm{E}$ O'Toole, ${ }^{1,2} \mathrm{~W}$ Alazawi. ${ }^{1}$ The Blizard Institute, Queen Mary University of London, UK; ${ }^{2}$ The Liver Unit, Barts Health NHS Trust, London, UK; ${ }^{3}$ Department of Dermatology, Barts Health NHS Trust, London, UK

\subsection{6/gutjnl-2014-307263.402}

Introduction Elevated transaminases (e.g., ALT) are frequently identified in various medical specialties, but the significance of abnormalities is often unclear. Patients with psoriasis are at higher risk of abnormal ALT due to drug-induced liver toxicity and alcohol excess. Recent reports highlight the association of psoriasis with metabolic syndrome, and suggest that nonalcoholic fatty liver disease (NAFLD) is more common in patients with psoriasis compared to the general population. Our aim was to determine (1) the prevalence and extent of raised ALT in a psoriasis clinic, (2) the degree to which such abnormalities are investigated and (3) the common causes identified.

Methods We undertook a retrospective analysis of adult patients with psoriasis followed up at the Royal London Hospital between Aug-Nov 2013. Electronic records were searched for demographics, blood results, hepatological investigations, psoriasis treatments, metabolic risk factors (diabetes, dyslipidaemia, hypertension, obesity), and alcohol intake. Elevated ALT was defined as $>40 \mathrm{U} / \mathrm{L}$ for men, $>35 \mathrm{U} / \mathrm{L}$ for women. The FIB-4 score was calculated to estimate risk of fibrosis.

Results Electronic records for 200 patients with psoriasis were reviewed. 57\% $(\mathrm{n}=114)$ were male, median age 43 years (range 17-81). 48\% were Caucasian, 22\% Bangladeshi, 12\% Indian, 6\% Pakistani and 6\% Afro-Caribbean.

LFTs were performed in $80.5 \%$ of patients $(\mathrm{n}=161)$, of whom 37\% ( $=59)$ had abnormal ALT on $\geq 2$ occasions (mean peak ALT 91, SD 63). 9 of these patients were on medication associated with deranged LFTs (4 on acitretin; 5 on methotrexate with persistently raised PIIINP). A further 15 patients drank excess alcohol. 3 patients (5\% of 21 tested) had viral hepatitis (1 HBV, 2 HCV).

Of the remaining 32 with unexplained elevated ALT, only 8 patients had a liver ultrasound (25\%), of whom 4 had signs of NAFLD. In addition, it is probable that another 17 patients who had negative liver screens but not had an ultrasound also have NAFLD. Therefore the estimated prevalence of NAFLD among psoriasis patients with raised ALT is 36\% $(21 / 59)$. There was no significant difference in age, sex or ethnicity between this group and the total psoriasis population.

Sufficient data were available to calculate FIB-4 scores in 12 of these 21 patients. 2 had scores that suggest moderate or advanced fibrosis, and warrant further investigation.

Conclusion Patients with psoriasis often have LFTs measured. These are often abnormal but under-investigated despite potentially representing significant, treatable disease. We identified a gap between LFT testing and screening for liver diseases, particularly viral hepatitis and NAFLD. There is a need for evidencebased guidance on investigation and referral in patients with deranged LFTs.

Disclosure of Interest None Declared.
PWE-143 ABNORMAL PLATELETS AND THE FORMATION OF ACTIVATED NEUTROPHIL-PLATELET COMPLEXES FOLLOWING PLATELET ADMINISTRATION INDUCES NEUTROPHIL ACTIVATION AND RELEASE OF REACTIVE OXYGEN SPECIES IN LIVER CIRRHOSIS

JP Sturgeon*, GK Manakkat Vijay, Y Zhang, JM Ryan, W Bernal, DL Shawcross. Institute of Liver Studies and Transplantation, King's College London School of Medicine, London, UK

\subsection{6/gutjnl-2014-307263.403}

Introduction The susceptibility to developing infection is well recognised in cirrhosis and circulating neutrophil dysfunction including excessive production of reactive oxygen species (ROS) is a major contributor to innate immune paresis. Platelets also play a key role modulating inflammation by interacting with neutrophils, secreting inflammatory mediators and influencing phagocytosis/apoptosis. The aim of this study was to examine platelet-neutrophil interactions in relation to ROS production and following healthy platelet exposure in patients with liver cirrhosis.

Methods Neutrophil-platelet interactions were characterised in 7 patients (6M; mean age 54) (Child-Pugh 11-14) in a paired crossover study with 7 healthy controls (HC). Neutrophils and platelets were isolated separately and incubated alone and together ex-vivo in zero, 50:1 and 100:1 platelet:neutrophil ratios. Neutrophils were stained with anti-CD16-PE and antiCD11b-APC-Cy7 (macrophage-1 antigen) using flow cytometry. Platelets were stained with anti-CD41a-APC (glycoprotein IIb/ IIIa) and complexes were identified as staining for CD11b/ CD16/CD41a. Neutrophils were stimulated with phorbol myristate acetate which induces ROS production quantified by conversion of dihydrorhodamine-123 to rhodamine- 123 .

Results The addition of platelets to neutrophils (100:1) significantly reduced ROS production $(\mathrm{p}<0.01)$. HC platelets were significantly better at reducing ROS production than cirrhotic platelets $(\mathrm{p}<0.05)$. Neutrophil-platelet complex formation was significantly higher when HC platelets were added to unstimulated neutrophils than cirrhotic platelets (Graph 1) with a 3.3 fold increase in neutrophil endothelial adhesion capability

Conclusion Cirrhotic platelets have a reduced capability to reduce neutrophil priming and ROS production. However, paradoxically administration of healthy platelets increases neutrophilplatelet complex formation and neutrophil adhesion capabilities which may promote endothelial activation and susceptibility to infection.

Disclosure of Interest None Declared.

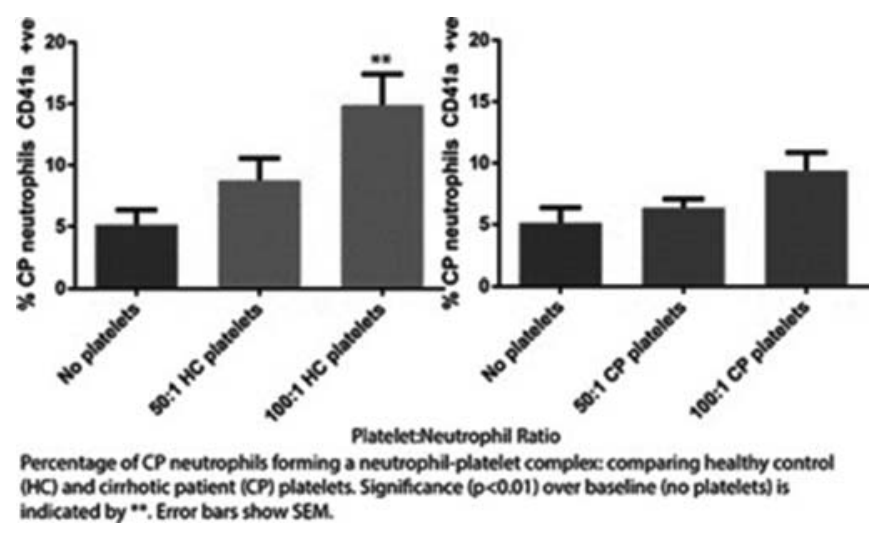

Abstract PWE-143 Figure 1 


\section{PWE-144 SMALL BOWEL CAPSULE ENDOSCOPY IN PATIENTS WITH CIRRHOSIS: THE EDINBURGH EXPERIENCE}

KJ Dabos*, A Koulaouzidis, PC Hayes, JN Plevris. The Royal Infirmary of Edinburgh, Edinburgh, UK

\subsection{6/gutjnl-2014-307263.404}

Introduction Portal hypertensive enteropathy (PHE) remains difficult to diagnose in patients with cirrhosis and portal hypertension. Limited test choices exist for the inspection of the small bowel in these patients. Small bowel capsule endoscopy (SBCE) would be ideal in this situation but it is rarely performed. ${ }^{1-3}$

Aim

We aimed to determine the prevalence of PHE using SBCE in a cirrhotic patient population from our centre.

Methods This was a retrospective study using the SBCE data base of our unit. We searched through 1,477 patients that had SBCE between 2005 and 2013. Patients with cirrhosis who underwent SBCE were identified, data retrieved and abstracted. The Fischer's exact or the chi-square tests were used to compare between groups. A two-tailed $P$ value of $<0.05$ was considered statistically significant.

Results We identified 53 patients with cirrhosis who underwent SCBE. We used PillCam ${ }^{\circledR}$ SB (Given ${ }^{\circledR}$ Imaging Ltd, Israel) system on 36 patients and the MiroCam ${ }^{\circledR}$ capsule (IntroMedic Co, Korea) on 17 patients. Thirty patients were referred for iron deficiency anaemia, 15 for obscure gastrointestinal bleeding, and 4 for other indications.

Four data sets were not available for review at the time of the study, leaving 49 patients to be reviewed. Mean age was 61.19 \pm 14.54 years $(\mathrm{M} / \mathrm{F}=27 / 22)$. Table 1 shows the aetiologies of liver disease in these patients. Six SBCE examinations were incomplete. Thirty three patients had evidence of portal hypertensive gastropathy (PHG) and 17 patients had evidence of oesophageal varices. In total, 29 patients had SCBE evidence of PHE (67\%). 28/29 (96.5\%) of patients with PHE had also evidence of PHG. 13/17 (76.4\%) patients with oesophageal varices had also evidence of PHE.

Our mean follow up was $58.0 \pm 13.7$ months. Twenty patients died during the follow up period. There was no correlation between the presence of PHE and aetiology of liver disease $(\mathrm{P}=0.4261)$ or subsequent death $(\mathrm{P}=0.2145)$.

Conclusion The prevalence of PHE in our study was $67 \%$. SBCE is a useful tool in evaluating PHE in cirrhotic patients irrespective of aetiology.

\begin{tabular}{ll} 
Abstract PWE-144 Table 1 & \\
\hline ALD & 15 \\
NAFLD & 9 \\
HepC & 7 \\
Cryptogenic & 6 \\
PBC & 6 \\
Other & 6 \\
\hline
\end{tabular}

\section{REFERENCES}

1 Rondonotti $\mathrm{E}$, et al. Capsule endoscopy in portal hypertension. Clin Liver Dis 2010;14:209-20.

2 Krystallis C, et al. Update of endoscopy in liver disease: more than just treating varices. World I Gastroenterol 2012;18:401-11

3 Sidhu $\mathrm{R}$, et al. Does small bowel capsule endoscopy alter management in patients with liver disease? Scand J Gastroenterol 2011:46:123-4

Disclosure of Interest None Declared.

\section{PWE-145 CHARACTERISATION OF CIRCULATING AND LIVER INFILTRATING MAIT CELLS IN HUMAN INFLAMMATORY LIVER DISEASES}

${ }^{1} \mathrm{~K}$ Parekh*, ${ }^{1} \mathrm{H}$ Jeffery, ${ }^{1} \mathrm{~J}$ Shaw, ${ }^{2} \mathrm{~T}$ Iqbal, ${ }^{1} \mathrm{DH}$ Adams, ${ }^{1} \mathrm{YH}$ Oo. ${ }^{1}$ Centre for Liver Research and NIHR BRU, University of Birmingham, UK; ${ }^{2}$ Gastroenterology, UHB NHS Foundation Trust, Birmingham, UK

\subsection{6/gutjnl-2014-307263.405}

Introduction Mucosal-Associated Invariant T (MAIT) cells are characterised by expression of the semi-invariant TCR $\alpha$-chain V $\alpha 7.2$ J $\alpha 33$ and high expression of CD161 and shown to play a role at mucosal barriers. They display a limited $\mathrm{T}$ cell receptor repertoire being restricted by the MHC class 1-related molecule, MR1 but secrete high levels of pro-inflammatory cytokines suggesting they may play an important role in liver inflammation. We have shown before that the majority of MAIT cells in circulation are CD8 ${ }^{+}$ MAIT cells. Recently, presence of MIAT cells have been described within human liver perfusate. However, very little is known about the phenotype and functions of liver infiltrating MAIT cells. In this study we investigated the frequencies and phenotypes of human liver infiltrating MAIT cells in healthy donors and diseased livers.

Methods Peripheral blood and explanted liver infiltrating lymphocytes were freshly isolated and phenotyped by multicolour flow cytometry. The MAIT population was defined as $\mathrm{CD} 3{ }^{+} \mathrm{CD} 16^{\mathrm{Hi}} \mathrm{Va} 7.2^{+}$

Results There was no difference in frequencies of circulating $\mathrm{CD} 3{ }^{\text {Pos }} \mathrm{CD} 161^{\mathrm{Hi}} \mathrm{Va} 7.2^{\text {Pos }}$ MAIT cells between patients with inflammatory liver disease and healthy controls $(1.4 \pm 0.7 \%$ vs. $2.3 \pm 1.0 \%)$ and the majority were $\mathrm{CD} 8^{\mathrm{Pos}}(82.3 \pm 3.1 \%)$ with a smaller population of $\mathrm{CD} 4{ }^{\mathrm{Pos}}(2.7 \pm 0.6 \%)$ and double negative $\mathrm{CD} 8{ }^{\mathrm{Neg}} \mathrm{CD} 4{ }^{\mathrm{Neg}}$ cells $(14.8 \pm 2.9 \%)$. Total CD $3{ }^{\mathrm{Pos}} \mathrm{CD} 161^{\mathrm{Hi}}$ Va7.2${ }^{\text {Pos }}$ MAIT frequencies were not significantly altered in inflamed liver tissue compared to blood $(4.4 \pm 1.0 \%$ vs. $1.4 \pm 0.7 \%)$. However, in the inflamed liver, the $\mathrm{CD} 8^{+}$subset was reduced $(61.2 \pm 6.2$ vs. $82.3 \pm 3.1, \mathrm{P}=0.006)$ while the $\mathrm{CD}^{+}$MAIT subset was increased $(15.9 \pm 5.6$ vs. $2.7 \pm 0.6, \mathrm{P}=0.02)$. CXCR3, liver homing chemokine receptor was highly enriched on circulating and liver infiltrating $\mathrm{CD} 3{ }^{\mathrm{Pos}} \mathrm{CD} 161^{\mathrm{Hi}}$ Va7. $2^{\mathrm{Pos}}$ MAIT cells $(>75 \%)$. Liver infiltrating MAIT cells expressed chemokine receptors CCR5 (78.4 \pm 7.2), CX3CR1 (51.3 \pm 10$)$, CCR6 (46.3 $\pm 14.8)$ and CXCR6 $(36 \pm 6.2 \%)$. Interestingly they expressed high levels of the integrin $\beta 7(39.1 \pm 3.6)$ and CD103 (19.6 \pm $5.5 \%)$, which are associated with mucosal immune responses. They also expressed the cytokine receptors IL23R $(27.1 \pm 8.5 \%)$ and IL18R $\alpha(76.7 \pm 5 \%)$.

Conclusion We have described for the first time that $\mathrm{CD} 3{ }^{\mathrm{Pos}} \mathrm{CD} 161^{\mathrm{Hi}} \mathrm{Va7} .2^{\text {Pos }}$ MAIT cells are present in inflamed human liver and express high levels of CXCR3 receptor implicated in lymphocyte recruitment to the liver and three other chemokine receptors CX3CR1 and CCR6 and CXCR6 that are associated with homing to portal tracts and bile ducts. Thus MAIT cells may play a role in biliary pathology. Disclosure of Interest None Declared.

\section{PWE-146 THE NEWCASTLE VARICES IN PBC (NVP) SCORE - A VALIDATION STUDY}

L Chinnappan*, T Cross, P Richardson, K Martin, I Patanwala. Department of Gastroenterology, Royal Liverpool University Hospital NHS Trust, Liverpool, UK

\subsection{6/gutjnl-2014-307263.406}

Introduction The NVP score ${ }^{1}$ is a simple, non- invasive externally validated tool which aims to predict variceal risk in patients 